\section{Case Reports in Ophthalmology}

\title{
Spironolactone for Secondary Central Serous Chorioretinopathy: A Challenge-Rechallenge Case
}

\author{
Kapil G. Kapoor ${ }^{a, b}$ Jennifer Sim ${ }^{b}$ \\ ${ }^{a}$ Wagner Macula and Retina Center, Virginia Beach, VA, USA; ${ }^{b}$ Eastern Virginia Medical \\ School, Norfolk, VA, USA
}

\section{Keywords}

Central serous chorioretinopathy $\cdot$ Spironolactone $\cdot$ Corticosteroids

\begin{abstract}
Central serous chorioretinopathy (CSCR) is potentially sight-threatening and has been associated with corticosteroid use. CSCR secondary to steroid use can sometimes be challenging to treat, especially if continuing steroid use is medically necessary. In this case report we demonstrate the efficacy of spironolactone as an effective agent in countering CSCR secondary to steroid use. This challenge-rechallenge case may be helpful to clinicians in delineating a treatment paradigm for these patients.

(C) 2017 The Author(s)

Published by S. Karger AG, Basel
\end{abstract}

\section{Introduction}

Central serous chorioretinopathy (CSCR) is a potentially sight-threatening condition with a complex pathogenesis that up to this time has defied precise elucidation. It is characterized by choroidal vascular dilation, increased choroidal thickness (pachychoroid), and subretinal fluid accumulation, and linked with endogenous and exogenous corticosteroid exposure $[1,2]$.

Up to half of patients have either a recurrent or chronic course ( $>3$ months), increasing the risk for visual morbidity secondary to retinal pigment epithelial atrophy or choroidal 
neovascularization [3]. Despite this, definitive treatment has remained particularly elusive. Recently, overaction of mineralocorticoid receptor (MR) pathways in choroidal vessels has been implicated in the pathophysiology of CSCR. Glucocorticoids also have affinity for MRs, further suggestive of a targeted role for the MR pathway in this condition [4]. A study in rat eyes demonstrated similar choroidal vascular dilation and leakage after activating the glucocorticoid and mineralocorticoid pathways with corticosterone and aldosterone, respectively [4].

Isolated small reports have described the use of MR antagonists clinically. One case report described subretinal fluid resolution in a patient with CSCR 6 weeks following treatment with eplerenone $25 \mathrm{mg} /$ day, with recurrence of subretinal fluid 2 months after discontinuation of eplerenone [5].

Another report described subretinal fluid resolution in 2 patients with chronic CSCR 5 weeks following treatment with oral eplerenone $25 \mathrm{mg} /$ day, with no recurrence for 5 months [6]. A subsequent pilot study evaluated 13 patients with chronic CSCR of at least 4months' duration. They were started on $25 \mathrm{mg} /$ day of eplerenone, which was increased to $50 \mathrm{mg} /$ day, and improvements in vision and OCT were noted [6]. More recently, reports have described the efficacy of spironolactone for CSCR [7-9], with one report in particular being a challenge-rechallenge case in a type A patient with no definitive evidence of a secondary cause [9]. These reports have helped build a database suggestive of the role of different MR antagonists having efficacy in CSCR.

It is important to remember that systemic corticosteroid use is an independent risk factor that has been associated with occurrences, prolongation, exacerbation, and recurrences of CSCR [7]. The mechanism in the paradoxical proedematous and subretinal fluid accumulation effects of glucocorticoids in CSCR pathogenesis stems from the aldosterone/MR pathway [10]. When corticosteroid use is involved, the typical treatment regimen is to avoid exogenous steroids when clinically possible. In this case, the patient required repeated steroid injections in the back to continue with activities of daily living and steroid avoidance was not possible. In cases of continued steroid exposure, CSCR management can prove quite challenging.

Though previous case reports have been published for the support of MR antagonists as a treatment option for chronic CSCR, this case is a unique contribution to the literature because case reports on CSCR thus far have focused on recalcitrant or persistent CSCR patients. In this case report we discuss a novel management regimen for a patient with recurrent, secondary CSCR due to steroid use.

\section{Case Report}

A 75-year-old Caucasian female presented to our clinic with 5 months of decreasing central vision with floaters in her left eye status after cataract surgery. Her visual acuity at that visit was OD 20/30 and OS 20/50-2 PHNI. Her past medical history was significant for 3 recent steroid injections in her back.

Her OCT revealed a pachychoroid in both eyes, with the left eye revealing foveal subretinal fluid with shaggy photoreceptors and a small pigment epithelial detachment (Fig. 1). There was no evidence of macular edema or subretinal hemorrhage on examination. Fluorescein angiography revealed an expansile dot of leakage. In concordance with the above findings, a diagnosis of CSCR secondary to her steroid injections was favored. An initial conversation of the condition, prognosis, and need for follow-up care was discussed. A course of 
oral spironolactone $50 \mathrm{mg}$ p.o. b.i.d. was initiated for presumed CSCR secondary to her steroid injections given 1 month previously. She was advised to avoid systemic steroid use if possible and to return for follow-up in 1 month. After 1 month, subretinal fluid was regressing only slightly, so the dose was increased to $50 \mathrm{mg}$ p.o. t.i.d. (Fig. 2). One month later, all subretinal fluid had resolved. Visual acuity had improved to 20/20. (Fig. 3)

Due to improvements, the patient was slowly tapered off spironolactone to $50 \mathrm{mg}$ p.o. b.i.d. with 1 month of follow-up. On the next follow-up, she continued to remain stable, so the spironolactone was tapered to $25 \mathrm{mg}$ p.o. b.i.d.. On the subsequent follow-up, she was tapered to $25 \mathrm{mg}$ p.o. daily with intent to stop spironolactone if continuously stable on the next follow-up. At the following follow-up she had a recurrence of subretinal fluid with an associated pigment epithelial detachment, which was also 3 weeks following another steroid injection in her back (Fig. 4). Her spironolactone was increased to $50 \mathrm{mg}$ p.o. b.i.d. again and the subretinal fluid regressed 2 months later. She was again tapered to $25 \mathrm{mg}$ p.o. b.i.d. and maintained on this maintenance dose.

It was apparent from her debilitating back condition that she would need steroid injections in her back approximately every 6 months, so the decision was made not to further taper her dose. She was monitored every 2 months following these injections for over a year and despite getting 2 more steroid injections in this time period, she had no recurrent subretinal fluid. Given her interim stability, the decision was made to conduct a retrial of spironolactone taper to $25 \mathrm{mg}$ p.o. daily. Three weeks later she presented for a nonscheduled appointment for acute metamorphopsia and had recurrent subretinal fluid with a pigment epithelial detachment. Given that this was approximately 6 months after her last steroid injection, it was thought to be related to a taper of her spironolactone maintenance dose rather than an acute response to a steroid injection as she had previously. Her spironolactone dose was increased to $25 \mathrm{mg}$ p.o. b.i.d., and 1 month later her metamorphopsia and subretinal fluid had resolved. Given this recurrence and her overall tolerance of the $25 \mathrm{mg}$ p.o. b.i.d. dosage, she was maintained on this dose with no recurrence for the ensuing 6 months.

\section{Conclusion}

Increasing evidence supports the increased risk of exogenous steroids with CSCR. When corticosteroid avoidance is not possible, this can lead to recalcitrant or recurrent CSCR with potential visual loss. This patient required ongoing steroid use to maintain her activities of daily living, and her CSCR recurrences were successfully managed with spironolactone. This challenge-rechallenge case shows definitively that spironolactone was therapeutic in dehydrating the subretinal space and inducing remission in this patient's secondary CSCR. This case may be helpful to clinicians in delineating a treatment paradigm for managing these patients. To our knowledge, this is the first case of treating a patient with secondary CSCR with spironolactone in a challenge-rechallenge case. This case demonstrates an avenue for managing patients who require steroids for maintaining activities of daily living and develop secondary CSCR in response to this. Titrating spironolactone doses judiciously and safely in response to this steroid-induced CSCR can be an important aspect of this therapeutic regimen. Further research is needed in randomized controlled trials to better understand the precise mechanism. 


\section{Statement of Ethics}

The authors confirm that the subject has given informed consent and that the study protocol has been approved by the institute's committee on human research.

\section{Disclosure Statement}

The authors have no disclosures.

\section{References}

1 Warrow DJ, Hoang QV, Freund KB: Pachychoroid pigment epitheliopathy. Retina 2013;33:1659-1672

2 Gemenetzi M, De Salvo G, Lotery AJ: Central serous chorioretinopathy: an update on pathogenesis and treatment. Eye 2010;24:1743-1756.

-3 Nicholson B, Noble J, Forooghian F, Meyerle C: Central serous chorioretinopathy: update on pathophysiology and treatment. Surv Ophthalmol 2013;58:103-126.

-4 Zhao M, et al: Mineralocorticoid receptor is involved in rat and human ocular chorioretinopathy. J Clin Invest 2012;122:2672-2679.

5 Gruszka A: Potential involvement of mineralocorticoid receptor activation in the pathogenesis of central serous chorioretinopathy: case report. Eur Rev Med Pharmacol Sci 2013;17:1369-1373.

-6 Bousquet E, et al: Mineralocorticoid receptor antagonism in the treatment of chronic central serous chorioretinopathy: a pilot study. Retina 2013;33:2096-2102.

7 Bousquet E, Beydoun T, Rothschild P-R, et al: Spironolactone for nonresolving central serous chorioretinopathy: a randomized controlled crossover study. Retina 2015;35:2505-2515.

8 Herold TR, Prause K, Wolf A, Mayer WJ, Ulbig MW: Spironolactone in the treatment of central serous chorioretinopathy - a case series. Graefes Arch Clin Exp Ophthalmol 2014;252:1985-1991.

-9 Ryan EH, Pulido CM: Central serous chorioretinopathy treatment with spironolactone: a challengerechallenge case. Retin Cases Brief Rep. 2015;9:235-238.

10 Daruich A, Matet A, Dirani A, Bousquet E, Zhao M, Farman N, et al: Central serous chorioretinopa thy: Recent findings and new physiopathology hypothesis. Prog Retin Eye Res 2015;48:82-118.

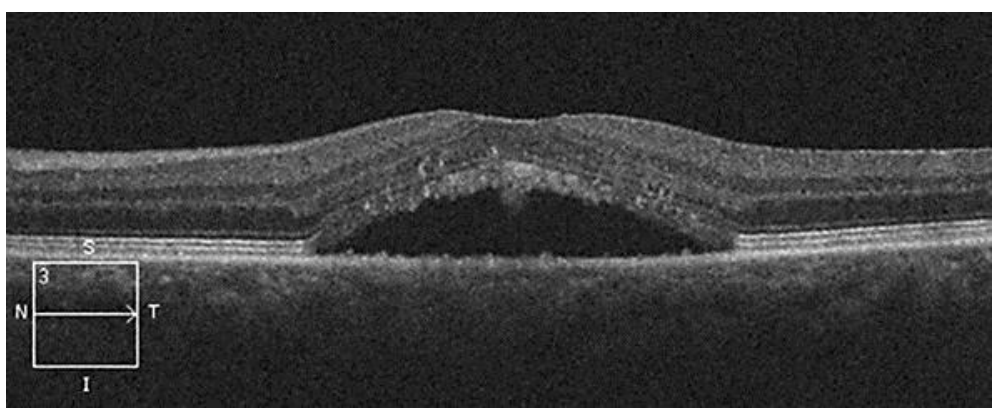

Fig. 1. OCT reveals subfoveal subretinal fluid and shaggy photoreceptors. 
Case Reports in
Ophthalmology

Case Rep Ophthalmol 2017;8:370-374

DOI: $10.1159 / 000477757$

C 2017 The Author(s). Published by S. Karger AG, Basel www.karger.com/cop

Kapoor and Sim: Spironolactone for Secondary Central Serous Chorioretinopathy: A Challenge-Rechallenge Case

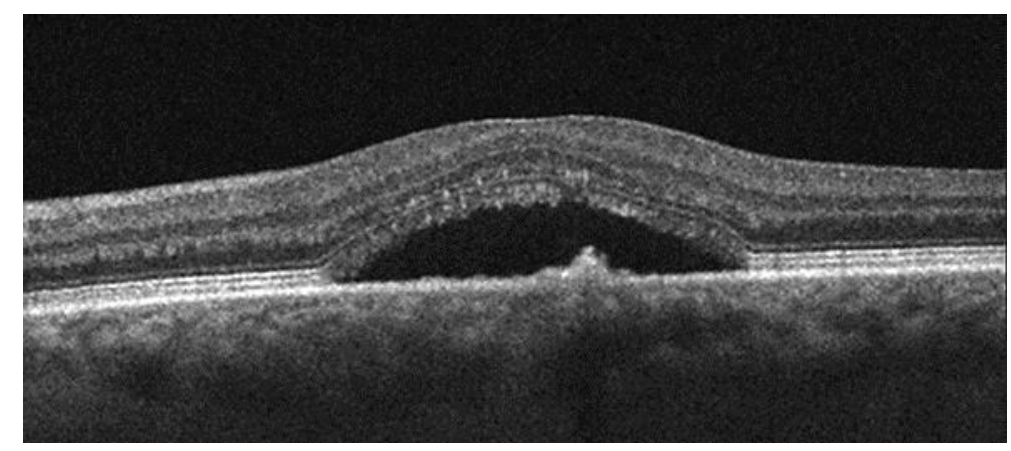

Fig. 2. The image depicts a slight reduction in subretinal fluid compared to Figure 1.

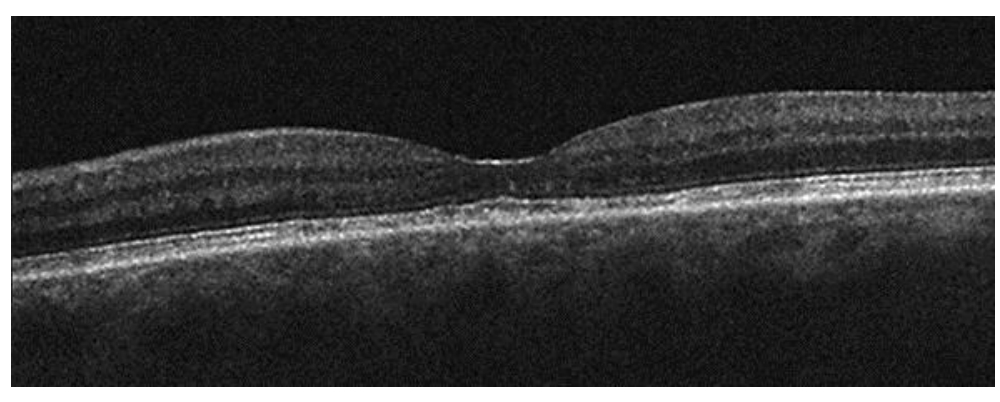

Fig. 3. A dry retinal contour is revealed with some retinal pigment epithelial stippling from previous central serous chorioretinopathy activity.

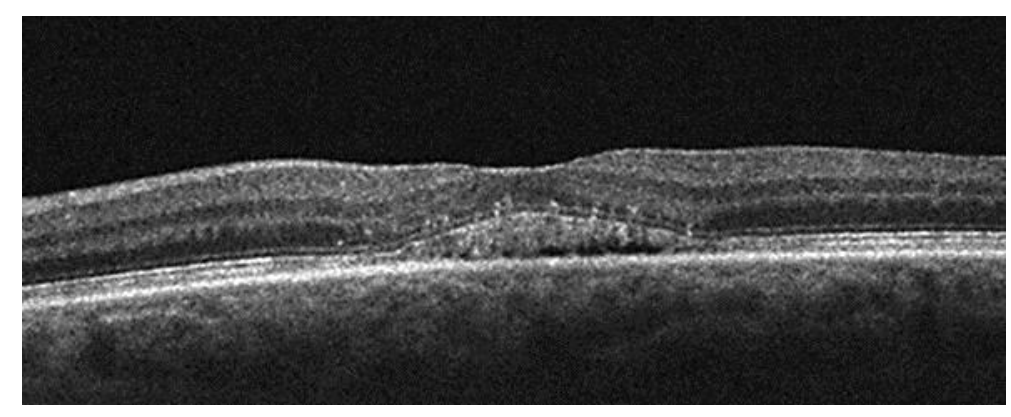

Fig. 4. The image reveals a slight recurrence of subretinal fluid. 